Iván González-Díaz, Manuel de-Frutos-López, Sergio Sanz-Rodríguez, Fernando Díaz-de-María

\title{
Adaptive multi-pattern fast block-matching algorithm based on Motion Classification techniques
}

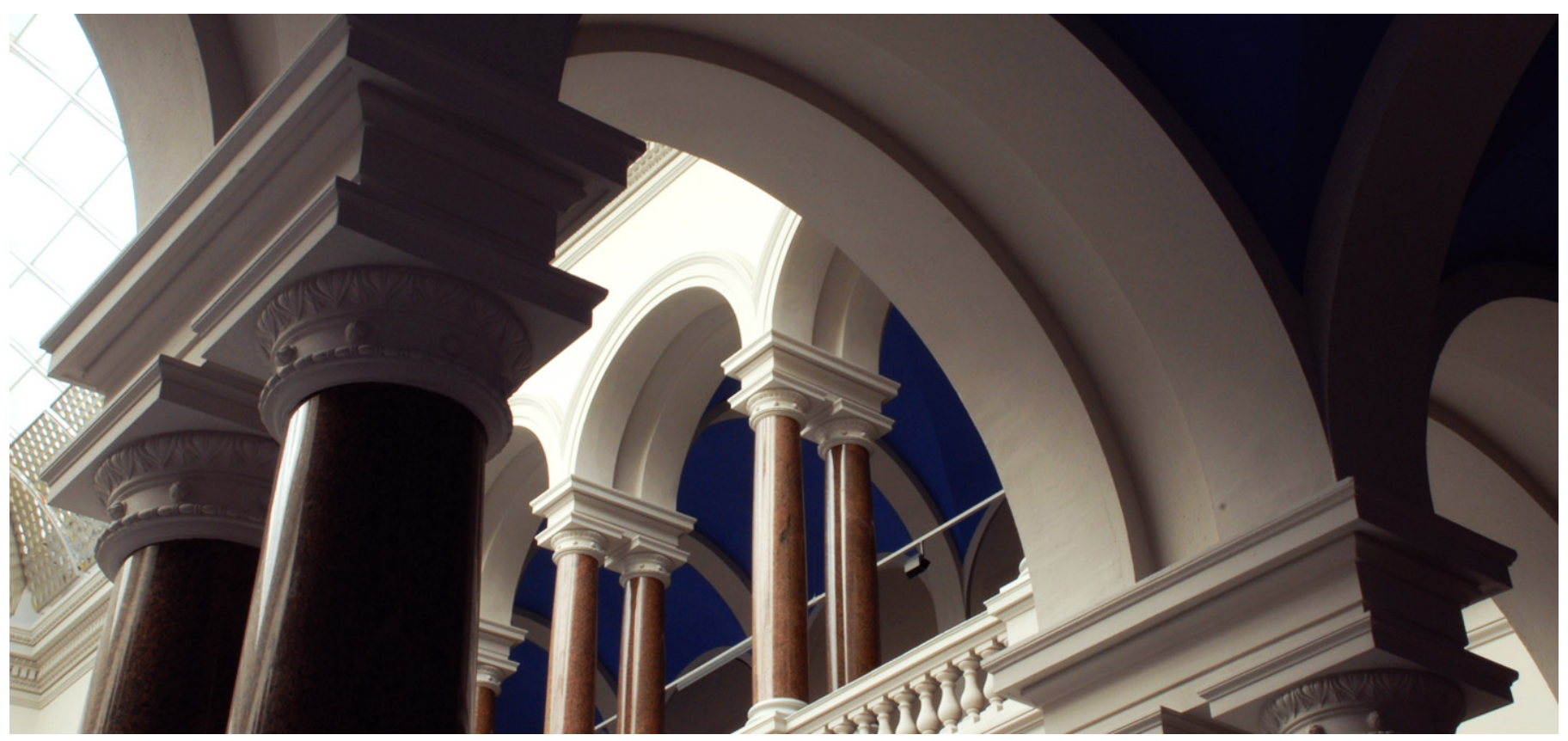

\section{Suggested Citation}

González-Díaz, Iván; de-Frutos-López, Manuel; Sanz-Rodríguez, Sergio; Díaz-de-María, Fernando: Adaptive multi-pattern fast block-matching algorithm based on Motion Classification techniques. - In: 2007 IEEE International Conference on Acoustics, Speech and Signal Processing : ICASSP. - New York, NY [u.a.] : IEEE, 2007. - ISBN: 1-4244-0727-3, pp. I.1177-I.1180. - DOI: 10.1109/ICASSP.2007.366123. (Postprint version is cited, page numbers differ.) 


\title{
ADAPTIVE MULTI-PATTERN FAST BLOCK-MATCHING ALGORITHM BASED ON MOTION CLASSIFICATION TECHNIQUES
}

\author{
Iván González-Díaz, Manuel de-Frutos-López, Sergio Sanz-Rodríguez, Fernando Díaz-de-María \\ Department of Signal Theory and Communications \\ Universidad Carlos III, Leganés (Madrid), Spain
}

\begin{abstract}
Motion estimation is the most time-consuming subsystem in a video codec. Thus, more eff cient methods of motion estimation should be investigated. Real video sequences usually exhibit a wide-range of motion content as well as different degrees of detail, which become particularly diff cult to manage by typical block-matching algorithms. Recent developments in the area of motion estimation have focused on the adaptation to video contents. Adaptive thresholds and multi-pattern search algorithms have shown to achieve good performance when they success to adjust to motion characteristics. This paper proposes an adaptive algorithm, called MCS, that makes use of an especially tailored classif er that detects some motion cues and chooses the search pattern that best $\mathrm{f}$ ts to them. Specif cally, a hierarchical structure of binary linear classif ers is proposed. Our experimental results show that MCS notably reduces the computational cost with respect to an state-of-the-art method while maintaining the quality.
\end{abstract}

Index Terms - Block-matching, motion estimation, motion classif cation, binary linear classif er

\section{INTRODUCTION}

Motion estimation is the most time-consuming subsystem in a video codec. Thus, more eff cient methods of motion estimation should be investigated. In this paper we deal with this problem focusing on the emerging H.264 standard, that has shown an excellent behaviour in a broad range of coding applications. Furthermore, H.264 incorporates new features such as the variable-size block estimation or multi-reference coding that make the motion estimation more effective but also more time-consuming.

Most recent developments in the area of motion estimation have focused on the adaptation to video contents. Real video sequences contain a wide-range of motion contents, distributed along spatial regions whose shape and position change over time. Recognizing these features can help with the selection of appropriate search patterns that minimize both the number of search points and the distortion.

Initial research on block-matching adaptation made special emphasis on the generation of adaptive thresholds: in [1] accumulated costs of previous blocks on the frame are used to generate adaptive thresholds that lead the search process; in [2] additional spatial regionalization is applied by computing costs of four spatial neighbours;

The authors would like to thank David Muñoz-Mejías and José Carlos Pujol-Alcolado for their inestimable help. however, the usefulness of these thresholds is still limited to the detection of early stops during the search process. Other developments, as in [3], employ various search patterns and select the most appropriate based on motion classif cation techniques; the main drawback of these techniques is that motion classif cation strongly depends on previous search results; thus, local minima may affect the next pattern selections, potentially inducing new errors on next blocks. In [4] this dependence is weaker, since the classif cation method uses a dispersion measure over the local vector feld. Even when a local minimum occurs, dispersion values can be large enough to select more exhaustive patterns in next blocks. Anyway, this measure by itself may not be robust enough to successfully classify every case. Our proposal uses a complete classif cation scheme that selects the most appropriate search pattern based on estimated motion characteristics. The objective of this scheme is to reduce computational complexity on those cases where light patterns can reach the optimal solution, while maintaining more exhaustive search patterns when needed.

The remainder of this paper is organized as follows. The details of our motion classif cation method are given in Section 2. A description of the average cost map used in the algorithm is provided in Section 3. Section 4 contains a performance comparison between our algorithm and another solution that has demonstrated good eff ciency at any bitrate. Finally, Section 5 summarizes our conclusions and outlines further work.

\section{MOTION CLASSIFICATION-BASED SEARCH (MCS)}

The Motion Classif cation-based Search (MCS) chooses among several heterogeneous search patterns one that best $\mathrm{f}$ ts particular motion contents. In particular, we pose this search pattern selection as a multi-class recognition problem that relies on some information available at coding time in order to characterise the motion and to select the search pattern that seems to be more robust and eff cient on each case. As in any classif cation problem, we need to carefully def ne the inputs (parameters), the outputs (classes) and the classif er structure.

\subsection{Local motion vector predictions}

Motion vector (MV) predictions are used as potential starting points of posterior ref nements of the local search. The H.264 standard def nes a median-based prediction from the MVs of spatially adjacent blocks. However, this prediction can be insuff cient and usually other correlated vectors are included to minimize later unnecessary 
searches around the initial search point. Our particular prediction set also includes:

1. Compensated MV of the co-located block.

2. Compensated MVs of four neighbouring blocks (left, up, upleft, up-right) on their best reference frame.

3. Median of compensated neighbouring vectors.

4. Uplayer vector (that resulting from the motion estimation performed for the next larger block size in the same reference).

5. Vector $(0,0)$.

Vector compensation scales vector magnitudes when they have been coded using different time distances (in visualization order) between the reference frame and the current frame. This process is explained in detail in [5].

\subsection{Problem parameterization: selected input features}

The problem parameterization is a critical issue in any classif er design. Based on correlation estimates between potential parameters and desired outputs, we have chosen nine inputs to be considered by our classif er, namely:

1. Block size: expressed in terms of the number of $4 \times 4$ blocks ( 1 for $4 \times 4$ to 16 for $16 \times 16$ ).

2. Binary input for homogeneous neighbourhood: when the four spatial neighbouring blocks (left, up, up-left, up-right) have the same compensated MVs this input is set to 1 , otherwise is set to 0 .

3. Time distance: when the time distance (presentation order) between a frame and its reference is larger, the expected time correlation between them decreases and more exhaustive searches are needed.

4. Number of neighbouring INTRA blocks: except in borders, INTRA coded blocks may indicate non-uniform motion that has been impossible to detect. Detecting this situation can prevent from continuously falling in local minima.

5. Absolute cost $\left(\mathbf{C}_{\mathbf{a b s}}\right)$ : The cost is measured as a sum of a distortion term (SAD or Sum of Absolute Differences) and a term that refers to the differential coding of the MV (compund of a Lagrangian Term $\lambda$ and a difference in bits between the prediction and the $\mathrm{f}$ nal MV). The absolute cost of the prediction is linearly adapted to $16 \times 16$ block-size and, then, made independent from the $\lambda$ value by means of:

$$
C_{a b s}=C_{p r e d}-45 \lambda
$$

The value of $45 \lambda$ has been empirically obtained by observing the evolution of the costs with respect to $\lambda$.

6. Cost ratio $\left(\mathbf{C}_{\text {ratio }}\right)$ : a ratio between predicted $M V$ costs and the Average Cost Map that will be described in section 3. The prediction cost is linearly adapted to $16 \times 16$ block-size and, then, the ratio is calculated as:

$$
C_{\text {ratio }}^{i}=\frac{C_{p r e d}^{i}}{T_{a v g}^{n, i}}
$$

7. Prediction MV magnitude: large prediction vectors are less reliable and usually require more exhaustive search patterns. The magnitude is calculated as:

$$
M a g=\left|M V_{\text {pred }}(x)\right|+\left|M V_{\text {pred }}(y)\right|
$$

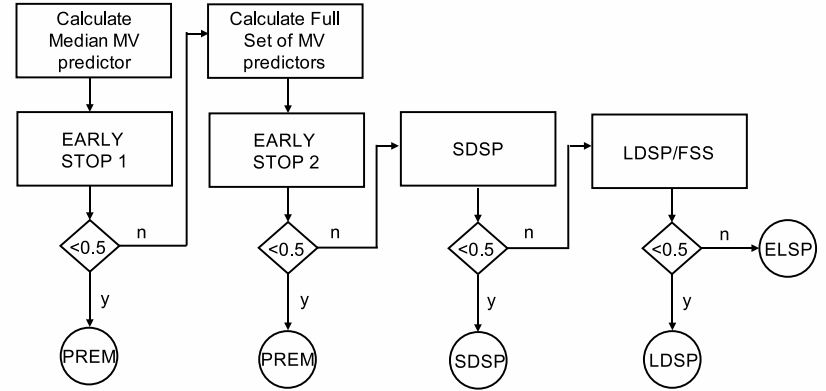

Fig. 1. Classif cation scheme of MCS. Early Stop 1 uses inputs $\{1,3,5,6,7,9\}$ while the rest of binary classif ers employ the complete input set

8. Motion Vector Dispersion (MVD): dispersion of temporal (co-located) and spatial (adjacent) correlated MVs can be useful to characterize the uniformity of the MV f eld of a block. Our dispersion measure obeys:

$$
\begin{array}{r}
D=\frac{1}{N} \sum_{i=1}^{N}\left(\left|M V_{i}(x)-M V_{\text {pred }}(x)\right|\right. \\
\left.+\left|M V_{i}(y)-M V_{\text {pred }}(y)\right|\right)
\end{array}
$$

9. Inverse of $\lambda\left(\lambda^{-1}\right)$ : In our experiments, $\lambda^{-1}$ has turned out to be more suitable to our purposes than $\lambda$.

\subsection{Classif cation outputs: search patterns}

In the MCS, the outputs of the classif cation are the search patterns described below:

1. Early Stop: the algorithm considers a prediction as the optimal solution and avoid any ref nement.

2. Small Diamond Search Pattern (SDSP): a diamond pattern with a 1 pixel step-size is used until a center point of one iteration is better than the other points.

3. Large Diamond Search Pattern (LDSP): a diamond pattern with a 2 pixels step-size is used until a center point of one iteration is better than the other points; afterwards, a ref nement is carried out by means of an SDSP pattern.

4. Exhaustive Logarithmic Search Pattern (ELSP): this pattern extends the well-known TSS [6] with a new stage. Specifically, it comprises 4 stages in which the step size decreases logarithmically starting from an initial step size of 8 pixels (sizes of $8,4,2,1$ ). This search pattern tries to emulate the functionality of the search range dependent predictor set presented in [5] and overcome diff cult situations such as those when predictions are far from the optimal solution or when the cost functions are not monotonic towards the optimal solution.

\subsection{Classif cation scheme}

Although the block-matching process is most time-consuming subsystem of the video codec, it is actually due to the high number of block-matching operations rather than to their individual cost. Therefore, the block-matching algorithm itself cannot incorporate complex and expensive classif ers that may take as much time as the 
posterior search patterns. Based on this assumption, we have chosen binary linear classifers as an example of light learning machines that can get accurate results in an eff cient manner. A binary linear classif er obeys:

$$
y=\mathbf{w}^{T} \mathbf{x}+b
$$

where $\mathbf{w}$ represents the vector of weights, $\mathbf{x}$ is the input vector with the parameter values (scaled between 0 and 1), $y$ is the output of the classif er and $b$ is the bias term. This classif er makes soft decisions that afterwards must be compared with a threshold ( 0.5 in our case), providing a hard decision ( 0 or 1$)$. We use several binary classif ers to solve the multi-class (multi-pattern) problem. In particular, we propose a hierarchical structure, illustrated in Fig. 1, that sequentially performs four binary classif cations. The output of each binary classif er is compared with a $\mathrm{x}$ threshold, so that smaller values imply a f nal classif cation decision and larger values lead to subsequent stages. Input values for Early Stop 1 classif er are related to H.264 standard median predictor. If an early stop is not detected, the full set of predictors is computed and the best initial vector is chosen, thus subsequent classif ers use updated information.

\subsection{Training and test sets generation}

In order to design every binary classif er, a training set and a test set (to evaluate the solution in terms of classif cation accuracy) have been generated by encoding typical video sequences for a thorough grid of QP values. These sets have been designed taking into account two main requirements: 1) they should be representative of the input space; and 2) they should make special emphasis on those samples in which selecting erroneous patterns produces unacceptable bitrate increases. Ignoring the second requirement leads to classif ers that always select classes with higher prior probability (early stops in our case). To meet both requirements, we assign to every sample a probability of being included on the training/test set according to the cost obtained by every potential search pattern for this particular sample (in order to compute this probabilities every pattern is tried for every sample):

$$
P=K\left(R+\frac{1}{C_{m i n}} \sum_{i=1}^{N}\left(C_{i}-C_{m i n}\right)\right)
$$

where $C_{i}$ is the cost achieved by the search pattern $i, N$ is the number of search patterns, $C_{\min }$ is the minimum cost (that obtained by the optimal search pattern), $R$ is a regularization term which ensures that any sample has a non-zero probability of being included on the set (even in early stops when $C_{i}=C_{\min }$ ), and $K$ is a function that manages the size of the training/test sets and guarantees that $0 \leq P \leq 1$.

\subsection{Classif ers training}

The training phase, i.e., the optimization of weights, is based on the minimization of the Mean Square Error (MSE) and LMS (Least Mean Squares) algorithm is used. However, it has not been possible to apply this procedure to every parameter. In particular, if we introduce $\lambda^{-1}$ on the optimization process, its corresponding weight turns out to be positive. This implies that light patterns are strengthened when working at lower qualities, what generates substantial bitrate increases. Therefore, we need to be more conservative (using more exhaustive patterns) at high QP values (high $\lambda$ values). To overcome this issue we have trained classif ers that use all input features except $\lambda^{-1}$ and, subsequently, got the optimal value of $\lambda^{-1}$ weight by means of a cross-validation process.

\subsection{Switching among search patterns}

Since our algorithm makes some classif cation errors, switches among search patterns should be considered for potential error detections. Although many potential switches have been tested, we have f nally included just one change between ELSP and SDSP, that is taken when at the end of the second step of the ELSP, the center is the best point and

$$
C^{i}<\left(\alpha+\frac{\beta}{\lambda}\right) T_{a v g}^{n, i}
$$

where $\alpha$ and $\beta$ have been empirically obtained. Again, when $\lambda$ decreases, switches to lighter patterns become more likely.

\section{AVERAGE COST MAP}

In order to achieve a good level of adaptation to several video features such as motion nature or background detail, we have developed an Average Cost Map (ACM). The ACM allows us to adapt the costs to the region contents (for example: high detailed regions usually imply high distortion values even when the block-matching process has reached the optimal solution). The averaging process is made in two separate domains: space and time. In space, an spatial average $S_{a v g}^{i}$ is made for each one macroblock-size region $i$ as a weighted arithmetic mean of the costs of the macroblock $i$ and its eight spatial neighbours:

$$
S_{a v g}^{i}=\alpha C_{i}+\frac{1-\alpha}{N} \sum_{j=1}^{N} C_{j}
$$

where $C_{i}$ is the accumulated cost of the MB $i, N=8$ represents the eight adjacent blocks, and $\alpha$ is a weighting factor $(0 \leq \alpha \leq 1)$ that establishes the relative importance of a block with respect to its neighbours. Then, a time average is performed as follows:

$$
T_{\text {avg }}^{n, i}=\beta S_{\text {avg }}^{i}+(1-\beta) T_{a v g}^{n-1, i}
$$

where $n$ is the time instant, $\beta$ is a weighting factor $(0 \leq \beta \leq 1)$ that establishes the relative importance of new data with respect to previous samples.

\section{EXPERIMENTAL RESULTS}

The proposed algorithm has been embedded in the H.264/AVC Reference Software Encoder (v10.2). Simulations have been made using IBBPBBP pattern, non-optimized RD (for real-time coding applications), search area of $33 \times 33,5$ references and exhaustive subpel ref nement. A thorough grid of QP values has been used in order to obtain results at bitrates very closed to those ones included on the Tables. The results at these particular bitrates have been obtained by linear interpolation.

Tables 1 and 2 show, respectively, PSNR vs bitrate and computational complexity (in terms of total search points per block and total coding time) comparison among Full Search (FS), full EPZS [5] (with extended diamond pattern and $\mathrm{fxed}$, temporal and spatial 
Table 1. Performance comparison (PSNR) among Full Search (FS), EPZS and MCS

\begin{tabular}{|c|c|c|c|c|c|c|c|}
\hline \multicolumn{8}{|c|}{ PSNR (dBs) vs Bitrate (Kbps) } \\
\hline & & 128 & 256 & 512 & $\mathbf{1 0 2 4}$ & 1536 & 2048 \\
\hline \multirow{3}{*}{ Mobile } & FS & 20.20 & 23.32 & 26.50 & 30.26 & 32.81 & 34.83 \\
\hline & EPZS & 20.32 & 23.26 & 26.47 & 30.25 & 32.82 & 34.84 \\
\hline & MCS & 20.30 & 23.25 & 26.47 & 30.24 & 32.79 & 34.82 \\
\hline \multirow{3}{*}{ Coastguard } & FS & 26.89 & 28.98 & 31.28 & 34.07 & 36.05 & 37.74 \\
\hline & EPZS & 26.91 & 28.99 & 31.28 & 34.08 & 36.09 & 37.79 \\
\hline & MCS & 26.90 & 28.97 & 31.26 & 34.07 & 36.10 & 37.81 \\
\hline \multirow{3}{*}{ Container } & FS & 34.34 & 37.22 & 40.17 & 43.12 & 44.95 & 46.28 \\
\hline & EPZS & 34.47 & 37.28 & 40.21 & 43.16 & 44.97 & 46.28 \\
\hline & MCS & 34.46 & 37.29 & 40.21 & 43.16 & 44.98 & 46.30 \\
\hline \multirow{3}{*}{ Tempete } & FS & 26.11 & 28.98 & 31.83 & 35.07 & 37.26 & 39.03 \\
\hline & EPZS & 26.21 & 29.00 & 31.82 & 35.06 & 37.27 & 39.05 \\
\hline & MCS & 26.21 & 28.99 & 31.81 & 35.06 & 37.28 & 39.05 \\
\hline
\end{tabular}

Table 2. Performance comparison (Search Points/Mean Coding Time per frame in seconds) among Full Search (FS), EPZS and MCS

\begin{tabular}{|c|c|c|c|}
\hline Sequence & FS & EPZS & MCS \\
\hline \hline Mobile & $1089 / 4.63$ & $10.99 / 0.80$ & $6.87 / 0.73$ \\
\hline Coastguard & $1089 / 6.44$ & $13.74 / 0.97$ & $7.74 / 0.85$ \\
\hline Container & $1089 / 4.83$ & $5.48 / 0.74$ & $1.38 / 0.64$ \\
\hline Tempete & $1089 / 5.08$ & $10.99 / 0.88$ & $4.65 / 0.76$ \\
\hline
\end{tabular}

memory predictors), and the proposed MCS algorithm. On the one hand, the FS is included just to provide reference PSNR vs bitrate results. On the other hand, the EPZS algorithm has shown good performance for a wide-range of motion contents and coding qualities, while it is computationally much more affordable than FS. However, it is still computationally expensive since it uses many predictors and search points with independence of the coding situation.

MCS results in PSNR (see Table 1) are really close to those achieved by EPZS and FS, with a mean loss of $0.01 d B s$ and a benef $t$ of $0.05 d B s$, respectively. Furthermore, PSNR losses do not increase at lower qualities, issue that becomes a classical problem for many block-matching algorithms. Poor FS results on low bitrates are due to the cost approximation used with the non-optimized RD coding option.

On the other hand, MCS algorithm achieves a mean computational complexity reduction (see Table 2 ) of $99.5 \%$ versus FS and $53.4 \%$ versus EPZS in mean search points per block. This reduction has an impact on the total coding time, with decreases of $86.6 \%$ versus FS and $12.3 \%$ vs EPZS. Complexity comparisons between EPZS and MCS are also provided in Figure 2.

\section{CONCLUSIONS AND FURTHER WORK}

The wide range of real-time video coding applications demands algorithms able to work at a wide range of qualities. MCS is intended to work at any coding quality as well as to follow different types of motion content. Motion classif cation techniques, which are the core of the MCS, can become the basis for the development of adaptive algorithms that fulf 1 these requirements. The reported results

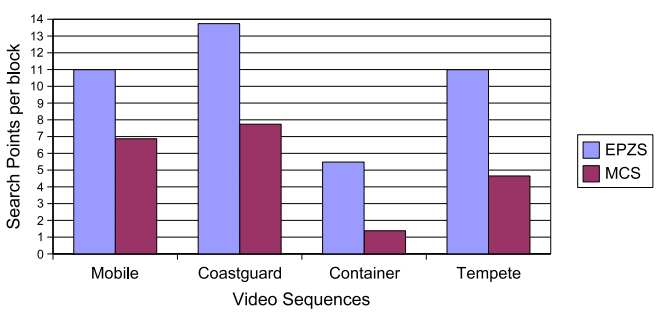

(a) Mean Search Points per block

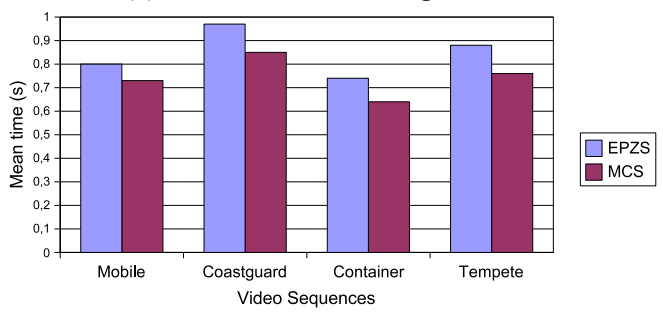

(b) Mean total coding time per frame

Fig. 2. Computational complexity comparison between EPZS and MCS in mean search points per block (a) and total encoding time (b)

show that MCS obtains similar quality values that known robust algorithms while notably reducing the computational complexity.

Further work mainly focuses on the development of different costs functions with two aims: the f rst one is to include $\lambda^{-1}$ in the training phase in the same way that the rest of the input features. The second is to make special emphasis on diff cult cases without modifying the prior probabilities of the classes.

\section{REFERENCES}

[1] Xiaoquan Yi and Nam Ling, "Improved partial distortion search algorithm for rapid block motion estimation via dualhalfway-stop," in IEEE International Conference on Acoustics, Speech, and Signal Processing, 2005. Proceedings. (ICASSP '05)., 2005, vol. 2, pp. ii/917-ii/920 Vol. 2.

[2] Jang-Jer Tsai and Hsin-Chia Chen, "Predictive block-matching discrepancy based rhombus pattern search for block motion estimation," in IEEE International Conference on Image Processing. ICIP 2005., 2005, vol. 1, pp. I-1073-6.

[3] Shih-Yu Huang, Chuan-Yu Cho, and Jia-Shung Wang, "Adaptive fast block-matching algorithm by switching search patterns for sequences with wide-range motion content," IEEE Transactions on Circuits and Systems for Video Technology, vol. 15, no. 11, pp. 1373-1384, 2005.

[4] I. Ahmad, Weiguo Zheng, Jiancong Luo, and Ming Liou, "A fast adaptive motion estimation algorithm," IEEE Transactions on Circuits and Systems for Video Technology, vol. 16, no. 3, pp. 420-438, 2006.

[5] A. M. Tourapis, "Fast me in the jm reference software," Joint Video Team (JVT) of ISO/IEC MPEG ITU-T VCEG (ISO/IEC JTC1/SC29/WG11 and ITU-T SG16 Q.6) 16th Meeting: 24-29 July 2005, Poznaǹ. JVT-P026.

[6] T. Koga, K. Iinuma, A. Hirano, Y. Iijima, and T. Ishiguro, "Motion compensated interframe coding for video conferencing.," in NTC, 1981, pp. vol. 4, pp G5.3.1-G5.3.5. 